\title{
Review on Recent Development and Application of Dispersive Liquid-Liquid Micro Extraction
}

\author{
ANBESSA TESHALE \\ ASMAMAW TAYE
}

\begin{abstract}
Dispersive liquid-liquid micro extraction (DLLME) has become a very popular environmentally benign samplepreparation technique, due to its simplicity, rapidity of operation and low consumption of solvent and reagent. It has attracted much interest from scientist working in separation science and much improvement has been made since its introduction in 2006. It has been combined with different extraction techniques such as floating organic drop, solid-phase extraction, and supercritical fluid extraction. This seminar has focused on the review on development of dispersive liquid micro extraction (DLLME) and its application in different samples, such as water, soil, food and biological material with different analytical techniques.
\end{abstract}

DOI: $10.7176 / \mathrm{CMR} / 11-3-02$

Publication date:March $31^{\text {st }} 2019$

\section{Introduction}

\subsection{Back ground of study}

In spite of substantial technological advances in analytical field, most instruments cannot directly handle complex sample matrixes yet. As a result, a sample-preparation step is commonly involved before instrumental analysis. The main aim of sample preparation is to clean up and concentrate the analytes of interest, while rendering them in a form that is compatible with the analytical system. Liquid-liquid extraction (LLE), based on the transfer of analyte from the aqueous sample to a water-immiscible solvent, is widely employed for sample preparation. Nevertheless, some short coming such as emulsion formation, use of large sample volumes and toxic organic solvents and hence, generation of large amounts of pollutants makes LLE labor to be intensive, expensive, timeconsuming and environmentally unfriendly. Another popular sample-preparation approach is solid-phase extraction (SPE). Although it uses much less solvent than LLE, the usage can still be considers significant, and normally an extra step of concentrating the extract down to a small volume was needed. SPE can be automating but this entails complexity and additional cost $[\mathbf{1 , 2}]$.

There have been substantial efforts in the past two decades to adapt the existing sample-preparation methods and develop new approaches to save time, labor and materials. So Miniaturization has been a key factor in the pursuit of these seminar review. Introduction of solid-phase micro extraction (SPME) [3] .Basically Initiated the interest for micro extraction techniques in analytical chemistry. With the SPME technique, target analytes of low or medium polarity are extracts from aqueous or gaseous samples onto a solid polymeric fiber. Extraction occurs by passive diffusion and the extraction yield essentially determined by the fiber to sample partition coefficient. It is a portable, simple to use relatively fast method and can be automated and coupled on-line to analytical instrumentation. However, the coated fibers are generally expensive, and for some applications, have limited lifetimes.

Liquid-phase micro extraction (LPME) as an alternative miniaturized sample-preparation approach emerged in the mid-to-late1990s $[4,5]$. As its name suggests, in LPME, only a micro liter volume of the solvent was needed to extract analytes from the aqueous samples. It overcomes many disadvantages of LLE as well as some of those of SPME (e.g. independence of a commercial supplier and sample carryover or cross-contamination) $[6,7]$.

Single drop micro extraction (SDME) was developed as a solvent-minimized sample pretreatment procedure. It is inexpensive, and since very little solvent is used, there is minimal exposure to toxic organic solvents [8,9] However, some disadvantages of this method are as follows fast stirring would tend to break up the organic drop, air bubble formation [10]. Extraction is time-consuming and equilibrium could not be attained after a long time in most cases [9]. As a solution to improve the stability and reliability of LPME, the alternative sample pretreatment procedure is hollow fiber liquid-phase micro extraction (HF-LPME) allows extraction and pre concentration of analytes from complex samples in a simple and inexpensive way. In general, the extraction efficiency achieved by HF-LPME is higher than direct-SDME, since hydrophobic hollow fibers allow the use of vigorous stirring rates to accelerate the extraction kinetics. Moreover, the use of hollow fibers provides protection of the extractants phase and hence, the analysis of dirty samples is feasible. Further, the small pore size of hollow fibers allows microfiltration of the samples, thus yielding very clean extracts [11]

Recent research has focused on the development of efficient, economical and miniaturized samplepreparation methods. Cloud point extraction (CPE) was based on phase separation, which occurs in aqueous solutions of non-ionic surfactants, when heated above the so-called cloud point temperature [12.] Besides, of the many benefits of CPE, the choice of surfactants often brings the nuisance to the analysis of analytes by analytical 
instruments such as GC and HPLC $[13, \mathbf{1 4}]$. In addition, the use of anionic surfactants as effective extractants in $\mathrm{CPE}$ often requires salts and adjustment of $\mathrm{pH}[\mathbf{1 5}, \mathbf{1 6}]$. Homogeneous liquid-liquid extraction (HLLE) utilizes the phase separation phenomenon from a homogeneous solution, and the target solutes are extracts into a sedimented phase. Ternary component solvent system and per fluorinated surfactant system are the two usual modes of HLLE [17-19]

Recently, a new mode of LPME based on solidification of floating organic droplet (LPME-SFO) was developed $[\mathbf{2 0}, \mathbf{2 1}]$. In this method, specific holders such as the needle tip of micro syringe, the hollow fiber and polychloroprene rubber (PCR) tube are not required for supporting the organic micro drop due to the use of organic solvents with low density and proper melting point. Combination of micro extracting systems and ultrasound (US) radiation provides an efficient pre concentration technique such as ultrasound-assisted emulsification-micro extraction (USAEME) for determining of analytes at trace levels. This pre concentration technique was first developed by. [22]The US radiation is an efficient tool to facilitate the emulsification phenomenon and accelerates the mass-transfer process between two immiscible phases, leading to an increment in the extraction efficiency of the technique in a minimum amount of time $[23,24]$.

[25] introduced dispersive liquid-liquid micro extraction (DLLME). is based on the ternary component solvent systems such as HLLE and CPE. It is a simple and fast micro extraction technique based on the use of an appropriate extractants, i.e., a few micro liters of an organic solvent such as chlorobenzene, chloroform or carbon disulfide with high density and a disperser solvent such as methanol, acetonitrile or acetone with high miscibility in both extractants and aqueous phases. When the mixture of extractants phase and disperser is rapidly injected into the sample, a high turbulence is produced. This turbulent regimen gives rise to the formation of small droplets, which are dispersed throughout the aqueous sample. Emulsified droplets have interfacial area. After the formation of cloudy solution, the surface area between the extracting solvent and the aqueous sample becomes very large, so the equilibrium state is achieved quickly and, therefore, the extraction time is very short.

In fact, this is the principal advantage of DLLME. After centrifugation of the cloudy solution, a sedimented phase is settled in the bottom of a conical tube and used with the most appropriate analytical technique. Other advantages of DLLME include simplicity of operation, rapidity, low cost, high recovery, high enrichment factor and environmental benignity $[\mathbf{2 5}, \mathbf{2 6}]$. This seminar review mainly focuses on the sample pretreatment, updated development, applications the publications related to the procedure, limitations and the further developments of DLLME will discussed.

\section{Review literature}

\subsection{Principles of DLLME}

DLLME consists of two steps: (1) Injection of an appropriate mixture of extracting and disperser solvents into aqueous sample, containing the analytes. In this step, the extracting solvent is dispersed into the aqueous sample as very fine droplets and the analytes are enriched into it. Owing to the large surface area between the extracting solvent and the aqueous sample, equilibrium state is achieved quickly and the extraction is independent of time. This is the most important advantage of this method. (2) Centrifugation of cloudy solution. After centrifugation, analytical instruments can determine analytes in the sedimented phase. The extraction steps of DLLME illustration are in Fig. 1. 

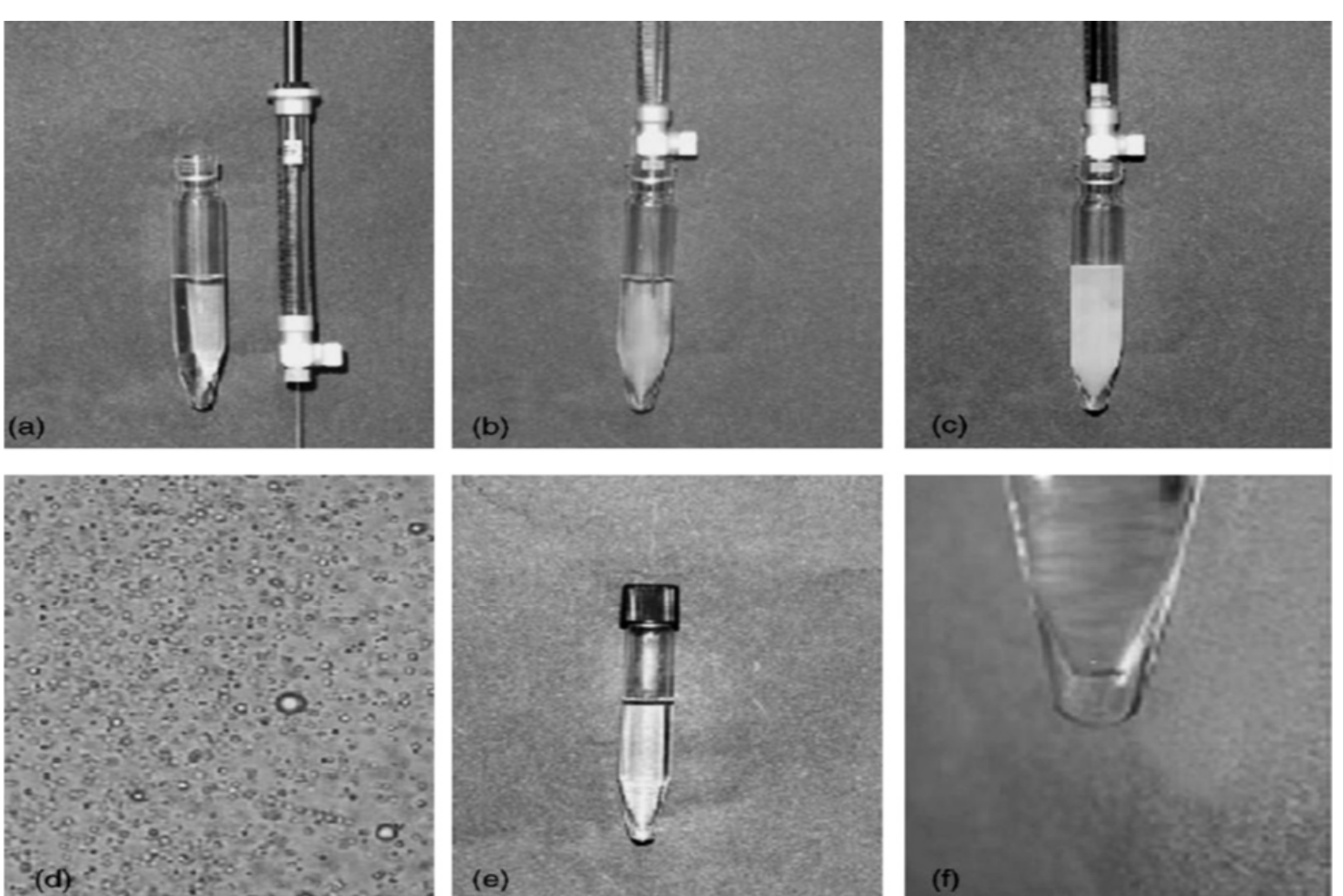

fig 1'Photography of different steps of DLLME: (a) before injection of mixture of disperser solvent (acetone) and extraction solvent (C2C14) into sample solution, (b) starting of injection, (c) end of injection, (d) optical microscopic photography, magnitude 1000 (that shows fine particles of $\mathrm{C} 2 \mathrm{Cl} 4$ in cloudy state), (e) after centrifuging and (f) enlarged view of sedimented phase (5.0 0.2_L). Reprinted with permission from [25]

\subsection{Development of DLLME}

\subsubsection{Conventional solvent based DLLM}

In DLLME, the factors that affect extraction efficiency are as follows: (1) suitable extracting solvent, (2) suitable disperser solvent,(3) volume of extracting solvent and (4) volume of disperser solvent(5) salt out effect and the PH effects. Selection of an appropriate extracting solvent is the major parameter for DLLME process. Organic solvents are selected based on their higher density rather than water, extraction capability of interested compounds and good chromatographic behavior. Halogenated hydrocarbons such as chlorobenzene, chloroform, carbon tetrachloride and tetra chloroethylene are usually selected as extracting solvents because of their high-density .Miscibility of disperser solvent in both extracting solvent and aqueous phase is essential in selection of it. Acetone, methanol and acetonitrile are usually selected as disperser solvents. since the number of organic solvents meeting this requirement is relatively small, and hazardous solvents. To overcome these various drawbacks, researchers have recently attempted to use solvents with a density lower than that of water and perform the extraction without using a disperser solvent which could possibly expand the applicability but it needs that of home-made vessels to pick up floating organic solvent [ 27].

To avoid the above disadvantage the selection of low density extraction solvent with appropriate amount and type of disperser solvent gives good extraction efficiency with relative low drawback[28] In DLLME The extracting solvent volume has important effect on the preconcentration factor $(\mathrm{PF})$. By increasing of the extracting solvent volume, the volume of sedimented phase obtained by centrifugation increases, resulting in a decrease on PF. Therefore, the optimal extracting solvent volume should ensure both high PFs and enough volume of the sedimented phase for the subsequent analysis after centrifugation. The disperser solvent volume directly affects the formation of cloudy solution (water/disperser solvent/extracting solvent), the dispersion degree of the extracting solvent in aqueous phase and, subsequently, the extraction efficiency. Variation of disperser solvent volume changes the volume of sedimented phase. Hence, it is necessary to change the volumes of disperser solvent and extracting solvent simultaneously to achieve a constant volume of sedimented phase. The suitable volume of disperser solvent for well cloudy solution depends on the volume of both aqueous phase and extracting solvent. In DLLME, the important factors affecting the volume of sedimented phase are:

(1) Solubility of extracting solvent in water,(2) sample solution volume,(3) disperser solvent volume and (4) 
extracting solvent volume. In experimental views, to obtain desired sedimented phase volume, some experimental tests should be done before the beginning of the main experiment. At first, solubility of extracting solvent in aqueous phase is calculated. Then, due to increase the solubility of extracting solvent in the presence of disperser solvent, some trial and errors should be done to calculate the exact volume of the sedimented phase that will be obtained using a desired volume of extracting and disperser solvents

In DLLME, extraction time is defined as an interval between the injection of mixture of a disperser solvent and extraction solvent, before centrifugation. The surface area between extraction solvent and aqueous phase is infinitely large. There by, transfer of analytes from aqueous phase to extraction phase is fast. Subsequently, equilibrium state is achieved quickly.

In DLLME, PF is defined as the ratio of the analyte concentration in the sedimented phase (Csed) and the initial concentration of analyte $(\mathrm{C} 0)$ in the sample:

$\mathrm{PF}=\mathrm{Csed} / \mathrm{C} 0$

Csed is obtained from a suitable calibration graph.

The extraction recovery (ER) is defined as the percentage of total analyte amount (n0), extracted to the sedimented phase (nsed):

$\mathrm{EF}=\frac{n \mathrm{sed}}{n o}=\frac{\text { csed } x V \operatorname{sed} x 100}{\operatorname{cox} v a q}$

Where Vsed and Vaq are the volumes of sedimented phase and sample solution, respectively.

The first DLLME procedure proposed by [29] required use of $1 \mathrm{~mL}$ of acetone as the dispersing solvent and $8 \mu \mathrm{L}$ of tetrachloroethylene as the extracting solvent for isolation of polycyclic aromatic hydrocarbons (PAHs) from $5 \mathrm{~mL}$ of water sample.[30 ]. This technology immediately attracted plenty attention of researchers in the world since it was published and then many papers on applications of DLLME to pre-concentration of organophosphorus pesticides, phthalate esters and chlorophenols and so on were published in next year's [31 ].

However, a few flaws of this approach appeared in the further applications in analyses of some compounds in different matrix. In order to promote the widespread applications of DLLME, substantial efforts have made to improve this procedure.

\subsubsection{Ionic Liquids Based on DLLME}

The traditional solvents used in DLLME not be considered as environmentally friend and the danger connected with toxic properties of chlorinated solvents led to the introduction of ionic liquids as the extracting solvents. Ionic liquids (IL), which consist of various organic cations and anions, are a new type of organic salt that have melting points less than or equal to $100^{\circ} \mathrm{C}$ and have favorable solvating properties for a range of polar and non-polar compounds. ILs are less hazardous than conventional organic solvents and are generally considered as green solvents because of their unique properties of high viscosity, negligible vapor pressure, excellent thermal stability, adjustable miscibility and polarity. Ionic liquids can be injected directly to high performance liquid chromatography (HPLC), although the high viscosity of compounds used led to the idea of diluting them before analysis [30]. Moreover, ionic liquids used in DLLME are heavier than water and are deposited on the bottom of the centrifuge tube. This makes it easier to handle them than long-chained alcohols, hydrocarbons and other solvents less dense than water [32] In recent years ionic liquids dispersive liquid-liquid micro extraction (ILDLLME) is more and more popular used due to its advantages described above. An environmentally friendly ILDLLME method coupled with HPLC for the determination of antihypertensive drugs irbesartan and valsartan in human urine samples was developed by. [33]. Six ionic liquids were tested for the first time as solvents in the extraction and preconcentration of deoxyribonucleic acid (DNA) using an in situ DLLME approach. The highest extraction efficiencies of DNA were obtained using 1-(1, 2-dihydroxypropyl)-3-hexadecylimidazolium bromide and N, N-didecyl-N-methyl-D-glucaminium bromide respectively. Extraction efficiencies higher than $97 \%$ were obtained using small amounts of ionic liquids $(0.50 \mathrm{mg})$ for each extraction [34].

In another study, a novel automatic on-line sequential injection dispersive liquid-liquid micro extraction method, based on 1-hexyl-3-methylimidazolium hexafluorophosphate ionic liquid as an extractants solvent was developed and demonstrated for trace thallium determination by flame atomic absorption spectrometry. The ionic liquid was on-line fully dispersed into the aqueous solution in a continuous flow format. No specific conditions like low temperature are required for extractants isolation. The results showed that for $15 \mathrm{~mL}$ of sample solution, an enhancement factor of 290 , a detection limit of $0.86 \mu \mathrm{g} / \mathrm{L}$ and a precision (RSD) of $2.7 \%$ at $20.0 \mu \mathrm{g} / \mathrm{L} \mathrm{Tl}(\mathrm{I})$ concentration level, were obtained. The developed method was evaluated by analyzing certified reference materials while good recoveries from environmental and biological samples proved that present method was competitive in practical applications [35]

It is not worthy that ionic liquids used in DLLME must be insoluble in water. This greatly reduces the list of extracting solvents. Typically, ionic liquids containing hexafluorophosphate ion are used, such as 1-butyl-3methylimidazolium hexafluorophosphate, 1-hexyl-3-methylimidazolium hexafluorophosphate, 1-octyl-3methylimidazolium hexafluorophosphate, 1-decyl-3-methylimida-zolium hexafluorophosphate, 1,3dibutylimidazolium hexafluorophosphate and 1-octyl-3-methylimida-zolium bis(trifluoromethylsulfonyl) 
imides[30] With the developments of IL-DLLME, more and more ionic liquids are attempted for being used as extracting solvents. Recently, 1,3-dipentylimidazolium hexafluorophosphate [PPIm][PF6] was used an alternative extractants in a study, where an approach was developed for the determination of a group of pesticides and metabolites (2-aminobenzimidazole, carbendazim/benomyl, thiabendazole, fuberidazole, carbaryl, 1-naphthol, and triazophos) from soils. A comparison of the performance of [PPIm][PF6] versus that of the so-common 1hexyl-3-methylimidazolium hexafluorophosphate ([HMIm][PF6]) was accomplished. Results indicate a comparable extraction efficiency with both ILs, being slightly higher with [HMIm][PF6] for the metabolite 2aminobenzimidazole, and slightly higher with [PPIm][PF6] for triazophos. In all cases, the LODs were in the low $\mathrm{ng} / \mathrm{g}$ range $(0.021 \mathrm{ng} / \mathrm{g}$ for [HMIm][PF6] and $0.026 \mathrm{ng} / \mathrm{g}$ for [PPIm][PF6]).

As a result, this study constitutes a starting point for the use of the IL [PPIm][PF6] for further analytical approaches.[ 36 ].The final precaution is that, as an alternative, sample heating was used occasionally in ILDLLME. Sometimes, the sample with added ionic liquid is heated until homogenous liquid is obtained. The ionic liquid droplets containing analytes are centrifuged after cooling down. This procedure is called temperaturecontrolled ionic liquid dispersive liquid-phase micro extraction (TC-IL-DLLME))[30] In a study, TC-IL-DLLME was introduced to analyze malachite green $(\mathrm{MG})$ and crystal violet $(\mathrm{CV})$ in environmental water by coupling with HPLC. In this method, 1-octyl-3-methylimidazolium hexafluorophosphate ([C8MIM][PF6]) and methanol were selected as appropriate extraction and dispersive solvents, respectively. Target compounds were extracted into the IL phase (dispersed completely in the aqueous phase) at a proper temperature. Under the optimum conditions, the established method offered good linear range $(0.25-20 \mu \mathrm{g} / \mathrm{L})$, low detection limits $(\mathrm{MG}, 0.086 \mu \mathrm{g} / \mathrm{L} ; \mathrm{CV}, 0.030$ $\mu \mathrm{g} / \mathrm{L}$ ), good reproducibility (relative standard deviation, $\mathrm{MG}, 9.4 \% ; \mathrm{CV}, 7.6 \% ; \mathrm{n}=5$ ), good recoveries $(91.7 \%$ for $\mathrm{MG}$ and $97.2 \%$ for $\mathrm{CV}$, respectively; $\mathrm{n}=5$ ) and high enrichment factor (254 for $\mathrm{MG}, 276$ for $\mathrm{CV}$ ) [ 37 ].

\subsubsection{DLLME Coupled with Auxiliary Instruments}

Though DLLME is a relatively rapid procedure compared with traditional extraction methods, efforts have given to make it coupled with some auxiliary instruments for improving its extraction efficient. Microwave and ultrasound are often used with DLLME. Besides, some other auxiliary instruments were attempted to couple with DLLME in recent several years.

\subsubsection{Microwave and ultrasound assisted DLLME}

Nowadays, microwave and ultrasound are main assisted methods used in DLLME. The high polarity of ILs results in them easily absorbing microwave energy, which can decrease the extraction time compared with conventional extraction methods. In a recent study, a simultaneous ionic liquid-based microwave-assisted dispersive liquidliquid micro extraction (IL-based MADLLME) method was developed for the determination of plasticizers in water using HPLC with ultraviolet detection. The parameters affecting the extraction efficiency, such as type and volume of ionic liquid and disperser solvent, microwave time and temperature were optimized

The optimal values were determined to be an extraction volume of $110 \mu \mathrm{L}$, a dispersive solvent volume of $0.26 \mathrm{~mL}$, and a microwave irradiation temperature and time of $60{ }^{\circ} \mathrm{C}$ and $2 \mathrm{~min}$, respectively. Under the optimum conditions, the limits of detection of these plasticizers were in the range of $0.71-1.94 \mu \mathrm{g} / \mathrm{L}$. Then validation of the methodology was carried out by the method of standard addition at two concentration levels for three water samples and the recoveries of the analytes were in the range of 85.2-103.3\%, with the relative standard deviations (RSDs, $\mathrm{n}=6$ ) $5.9 \%$. The results showed that IL-based MADLLME is a suitable method for the determination of these five plasticizers in water. An in-situ IL-based MADLLME followed by HPLC was developed for determination of the triazine herbicides ametryne, prometryne, terbuthylazine and terbutryn in water samples. The type and volume of IL, the type and volume of disperser, microwave irradiation temperature, extraction time and salt concentration were optimized. The results showed that the recoveries were $88.4-114 \%$. The relative standard deviations were in range of 1.6-6.2\%, and limits of detection were between 0.52 and $1.3 \mu \mathrm{g} /[\mathbf{3 8}]$.

It is well-known that ultrasound is a powerful aid in the acceleration of various steps in the processes of separation and extraction, such as emulsion forming, homogenizing and mass transferring between immiscible phases [ 39 ]. In recent years, ultrasound-assisted IL-based DLLME was developed and widely applied to analytical chemistry, which could obtain high extraction efficiency and extraction equilibrium in a very short time.

A sample preparation method, namely ionic liquid-based ultrasound-assisted dispersive liquid-liquid micro extraction (IL-USA-DLLME), followed by HPLC has been developed for the extraction and preconcentration of four benzophenone-type ultraviolet filters from three different water matrices. The ultrasound-assisted process was applied to accelerate the formation of the fine cloudy solution, which markedly increased the extraction efficiency and reduced the equilibrium time. In another study, IL-USA-DLLME procedure was developed for the extraction of eight fluoroquinolones including marbofloxacin, norfloxacin, ciprofloxacin, lomefloxacin, danofloxacin, enrofloxacin, oxolinicacid and nalidixic acid in groundwater, using high performance liquid chromatography with fluorescence detection (HPLC-FLD). The ultrasound-assisted process was applied to accelerate the formation of the fine cloudy solution using a small volume of disperser solvent $(0.4 \mathrm{~mL}$ of methanol). For the DLLME procedure, the IL 1-octyl-3-methylimidazolium hexafluorophosphate and methanol were used as extraction and disperser solvent, respectively. Under the optimum conditions, linearity of the method was observed over the range 
10-300 ng/L with correlation coefficient $>0.9981$. The proposed method has been found to have excellent sensitivity with limits of detection between 0.8 and $13 \mathrm{ng} / \mathrm{L}$ and precision with relative standard deviation values between 4.8 and 9.4\% $(n=5)$. The enrichment factors and recoveries were $122-205$ and $85-107 \%$, respectively. This simple and economic method has been successfully applied to analyse real groundwater samples with satisfactory results [41]

Moreover, IL-USA-DLLME combined with in-situ metathesis reaction was developed for the determination of five phenylurea pesticides (i.e., diuron, diflubenzuron, teflubenzuron, flufenoxuron, and chlorfluazuron) in environmental water samples. In the developed method, $360 \mu \mathrm{L} \mathrm{LiNTf2}$ aqueous solution $(0.162 \mathrm{~g} / \mathrm{mL})$ was added to the sample solution containing a small amount of [C6MIM]CI $(0.034 \mathrm{~g})$ to form a water-immiscible ionic liquid, [C6MIM]NTf2, as extraction solution. The mixed solutions placed in an ultrasonic water bath at $150 \mathrm{~W}$ for $4 \mathrm{~min}$ and centrifuged at $3500 \mathrm{rpm}$ for $10 \mathrm{~min}$ to achieve phase separation. The quantity of [C6MIM]CI, the molar ratio of [C6MIM]CI and LiNTf2, ionic strength, ultrasound time, and centrifugation time, were optimized. The optimized technique provides good repeatability (RSDs 2.4 to 3.5\%), great enrichment factor (244 to 268), linearity $(0.5$ to $500 \mu \mathrm{g} / \mathrm{L})$, and low limits of detection $(0.06$ to $0.08 \mu \mathrm{g} / \mathrm{L})$. The developed method can be applied in routine analysis for the determination of phenylurea pesticides in environmental sample.[ 42].

It should be pointed out that microwave and ultrasounds are not only used in IL-based DLLME but also in routine DLLME. Microwave-assisted extraction (MAE) coupled with DLLME followed by semi-automated insyringe back-extraction technique was used for extraction of five chlorophenols from soil and marine sediment samples [43 ] Microwave-assisted extraction was performed by using $2.0 \mathrm{~mL}$ of alkaline water at $\mathrm{pH} 10.0$. After extraction, the $\mathrm{pH}$ of extraction solution was adjusted at 6.0 and DLLME procedure was done using $1.0 \mathrm{~mL}$ of acetone as a disperser solvent and $37.0 \mu \mathrm{L}$ of chlorobenzene as extraction solvent. The obtained recovery and preconcentration factors for the analytes were in the range of $68.0 \%-82.0 \%$ and $25-30$, respectively, with relative standard deviations $7.6 \%$. The limits of the detection were found in the range of $0.0005-0.002 \mathrm{mg} / \mathrm{kg}$. The method provides a simple and fast procedure for the extraction and determination of chlorophenols in soil and marine sediment samples [43].

A simple and efficient method was developed using MAE-DLLME coupled with GC-MS for the extraction and quantification of 16 polycyclic aromatic hydrocarbons (PAHs) in smoked fish. Several parameters, including the types and volume of hydrolysis, extracting and disperser solvents, microwave time and $\mathrm{pH}$, were optimized. Under the optimum condition, the MAE-DLLME method coupled with GC-MS provided excellent enrichment factors (in the range of 244-373 for 16 PAHs) and good repeatability (with a relative standard deviation between 2.8 and $9 \%$ ) for spiked smoked fish. The calibration graphs were linear in the range of $1-200 \mathrm{ng} / \mathrm{g}$, with the square of the correlation coefficient (R2) $>0.981$ and detection limits between 0.11 and $0.43 \mathrm{ng} / \mathrm{g}$. The recoveries of those compounds in smoked fish were from $82.1 \%$ to $105.5 \%$. A comparison of this method with previous methods demonstrated that the proposed method is an accurate, rapid and reliable sample-pretreatment method that gives very good enrichment factors and detection limits for extracting and determining PAHs from smoked fish [ 44]

Moreover, a method based on MAE in combination with DLLME followed by gas chromatography-electron capture detection (GC-ECD) has proposed as a new approach for the sensitive determination of cork taint responsible compounds in cork stoppers and oak barrel sawdust. Under the optimal condition, the proposed method showed satisfactory linearity (correlation coefficients over 0.991 ), good repeatability (below 10.4\%) and inter-day precision (below 11.2\%). Detection limits obtained were similar or even lower than previously reported. The results obtained proved the suitability of MAE-DLLME as a sensitive sample preparation method for the analysis of haloanisoles and halo phenols in solid enological matrices.[ 45 ] Nowadays, an increasing number of papers concerned about the application of USA-DLLME in analytical chemistry have been published. In a recent study, USA-DLLME coupled with gas chromatography-flame ionization detector was developed for the determination of benzene, toluene and xylenes isomers (BTX) in water samples.

\subsubsection{Magnetic stirring-assisted and vortex assisted DLLME}

Lastly, magnetic stirring-assisted and vortex-assisted DLLME were reported. A method, namely magnetic stirringassisted DLLME followed by HPLC with ultraviolet detection, was developed for the determination of phthalate esters (PEs) in water samples. This novel microextraction method is based on the fast injection of extracting solvent into the aqueous solution, which is being stirred by a magnetic stirrer, to form a cloudy binary component solvent system. The extraction parameters such as type and volume of extracting solvent, $\mathrm{pH}$ of sample, salt addition, extraction time and stirring rate were optimized. The result showed that the optimal stirring rate is $1000 \mathrm{rpm}$. Under the optimal condition, the limits of detection and quantification ranged from 0.13 to $0.38 \mu \mathrm{g} / \mathrm{mL}$ and 0.43 to $1.27 \mu \mathrm{g} / \mathrm{mL}$, respectively. The ranges of intra-day and inter-day precisions $(\mathrm{n}=5)$ at $100 \mu \mathrm{g} / \mathrm{mL}$ of PEs were $1.50-2.65 \%$ and $2.31-3.35 \%$, respectively. The results indicated that MSA-DLLME method was successful for pre concentration of PEs in drinking and environmental water samples [44].

Moreover, vortex-assisted DLLME coupled with GC-MS has been developed and used for the analysis of six benzophenone ultraviolet filters in water samples. The obvious advantage of this new method is that no centrifugation and disperser solvent were required in this micro extraction procedure. Meanwhile, short extraction 
time and high extraction efficiency achieved. This method opens up a potentially new horizon for on-site dispersive liquid-liquid micro extraction. Under the optimum conditions, the proposed method provided good enrichment factors up to 310 , with relative standard deviations ranging from 6.1 to $12.9 \%$. The limits of quantification were in the range of 20-100 $\mathrm{ng} / \mathrm{L}$. The results showed that the proposed method was feasible for the determination of UV filters from spiked genuine water samples and acceptable recoveries over the range of 71.0-120.0\% were obtained [45].

\subsection{Combination of DLLME with Other Extraction Techniques 2.3.1 DLLME combined with SFO}

As shown above, the concerns connected with toxicity of chlorinated solvent led to use of less toxic solvents such as long-chained alcohols or hydrocarbons. These solvents are lighter than water and must be collected from the above surface of the water sample, so different methods of collecting the extracting solvent had to be invented [46 ]. In 2007, a new mode of liquid-phase micro extraction based on solidification of floating organic droplet (LPMESFO) was developed [52 ]. In this method, no specific holders such as the needle tip of micro syringe, hollow fiber or polychloroprene rubber tube is required for collecting organic micro drop rather easily by solidifying it at low temperature. However, the extraction time was somewhat long, thus it cannot satisfy the demand of fast analysis. Therefore, a new method based on DLLME and LPME-SFO was proposed, which overcomes the problems mentioned above. The large contact surface between the sample and the droplets of the extractants speeds up mass transfer, as fast as DLLME, and shorter extraction time than that of LPME-SFO. In DLLME-SFO, lower toxicity extracting solvents can be used and the floated extractants is solidified and easily collected from the top of the solution for analysis. As a result, with these merits, more and more papers on the applications of DLLME-SFO are published during past several years [ 30 ]

Recently, DLLME-SFO was applied to the simultaneous separation and preconcentration of iron in water samples [48] . For determining chlorpyrifos in environmental water samples, DLLME-SFO followed by GC with flame photometry detection has been adopted and improved [49]. A method for the analysis of 14 anilines in water samples by DLLME-SFO prior to GC-MS was developed and optimized [55] Furthermore, a novel surfactantassisted dispersive liquid-liquid microextraction (SADLLME) based on SFO combined with HPLC-UV has been proposed for extraction and determination of amphetamine and methamphetamine in urine samples. The application feasibility of SFO-SADLLME-HPLC-UV in real sample was investigated by analyzing different real samples and satisfactory results were obtained [50].

\subsubsection{DLLME combined with SPE}

SPE is a widely used sample preparation technique for the isolation of selected analytes, usually from a gas, fluid or liquid phases. The principal goals of SPE are trace enrichment (concentration), matrix simplification and medium exchange. However, DLLME is not suitable for complex matrices such as highly saline solution. So, a combination of SPE and DLLME was introduced by [51]. SPE-DLLME is an efficient hyphenated technique that offers the advantages of both methods such as simplicity, low solvent usage and exposure, low disposal cost and extraction time, with high recovery and enrichment factor, and it can be also used in complex matrices.

The SPE-DLLME was applied to the preconcentration and analysis of short-chained dodecyl alcohol ethoxylates and dodecyl alcohol. The results showed that the analytes were preconcentrated 700 times with the use of small sample volume. The developed method was used for the analysis of short-chained dodecyl alcohol ethoxylates and dodecyl alcohol in both sewage effluent from sewage treatment plants and river water samples [52 ]

\subsubsection{DLLME combined with SDME}

The single-drop microextraction (SDME) performed by suspending a micro liter drop of water-immiscible solvent in the stirred aqueous solution or placing the drop in the headspace of sample bottle. Its extreme simplicity motivates many successful applications. However, this technique suffers much from the instability of the suspending drop and approaching high signal intensity of analyte usually takes quite some time for extraction. The integration of these two techniques, i.e. DLLME and SDME is presented as a new extraction method for environmental analysis. This proposed process allows the individual short comings of each technology to be overcome and leads to an optimum process configuration [ 52]

Furthermore, DLLME is uses mainly for the pre-concentration of the analytes into an extraction organic solvent, which after wards using GC as detection system and SDME is used for the back extraction of analytes in the water samples, which can provide good situation for using HPLC analysis. Therefore, in a combination of these two methods, HPLC can be used as detection system. The combination of DLLME and SDME as a new preconcentration technique is developed for the separation and determination of acidic non-steroidal antiinflammatory pharmaceutical compounds, namely, naproxen, diclofenac, and ibuprofen, in water samples using HPLC-UV. Good linearity range of $0.11000 \mu \mathrm{g} / \mathrm{L}$, acceptable reproducibility's (RSDs, 4.5-8.8\%), low limits of detection $(0.03-0.2 \mu \mathrm{g} / \mathrm{L})$, and satisfactory relative recoveries were obtained.

Moreover, the low-density solvent-based DLLME combined with SDME was developed in a new format of 
fast three-phase micro extraction for the first time. In a study, a volume of low-density solvent (toluene) was used as organic phase and injected into the aqueous sample (donor phase) with disperser (methanol). The analytes were pre-extracted into the organic sample within $2 \mathrm{~min}$. Afterwards the layer of the organic phase was formed on the top of the aqueous phase by a 2 min centrifugation. Then a drop of acceptor solution was introduced into the upper layer of toluene and the SDME was carried out for back extraction. After extraction, the acceptor drop was with draw and directly injected in to a HPLC-UV for analysis. In this procedure, the high speed and efficiency of DLLME make the typical stirring step in SDME unnecessary and the total extraction time noticeably short [53 ]

\subsubsection{DLLME combined with SFE}

Supercritical fluid extraction (SFE) has been adopted to extract different substances from solid matrices since three decades ago. As is well known, in spite of substantial advantages of DLLME, it is not suitable for extraction of compounds from solid samples and some time-extra steps such as drying and filtering processes in sample preparation before DLLME are time-consuming. Also, sometimes it is impossible to do DLLME for the extraction of analytes from complex matrices [54] For the first time, a combination of SFE and DLLME, as a samplepreparation method was developed for determination of ten PAHs in marine sediment samples. In SFE-DLLME, the collecting solvents such as methanol and acetonitrile in SFE can be used as disperser solvent in DLLME. After performing SFE and collecting the extracted analytes in the disperser solvent, a suitable volume of the extracting solvent was added into the collecting solvent. Finally, the mixture was injected to the aqueous sample. The other steps were similar to DLLME method. SFE-DLLME leads to high pre concentration factor for determining organic compounds in solid samples, easy use of DLLME in solid samples and can eliminate the need to evaporate the collecting solvent at the end of SFE. The performance of SFE-DLLME in the extraction of polycyclic aromatic hydrocarbons (PAHs) from different marine sediment samples with various matrices was excellent. PAHs were employed as model compounds to assess the extraction procedure and were determined by gas chromatographyflame ionization detection (GC-FID). SFE of PAHs was performed at $313 \mathrm{~K}$ and 253.2 bar, at static and dynamic time 10 and $30 \mathrm{~min}$, respectively. The extracted PAHs were collected in $1 \mathrm{~mL}$ of acetonitrile. Subsequently, $16 \mu \mathrm{L}$ of chlorobenzene (as extraction solvent) was added to collecting solvent (1.0 mL of acetonitrile). Then, the mixture was injected rapidly into $5.0 \mathrm{~mL}$ of aqueous solution.After centrifugation, the PAHs in the sedimentated phase were analyzed by GC-FID. Under the optimum conditions, the calibration plots were linear in the range of $0.4-$ $41.6 \mathrm{mg} / \mathrm{kg}$ and the limits of detection were $0.2 \mathrm{mg} / \mathrm{kg}$ for all of the analytes. Analysis of PAHs in different solid samples showed that the improved technique has great potential for PAHs analysis in marine sediments [55].

\subsection{Applications of DLLME with instruments \\ 2.4.1. DLLME combined with GC}

Since water-immiscible solvents are generally uses in DLLME, this is preferred technique for the analysis of extracts in GC. The application of DLLME was developed for extraction and determination of polycyclic aromatic hydrocarbons (PAHs) in water sample [25]. One $\mathrm{mL}$ of acetone (as disperser solvent) containing 8.0_L of C2C14 (as extracting solvent) was rapidly injected into a $5.00 \mathrm{~mL}$ of the sample solution by a $1.00-\mathrm{mL}$ syringe, and the mixture was gently shaken. Then, the mixture was centrifuged and 2.00_L of the sedimented phase was injected into the GC for analysis. Under the optimum conditions, the obtained PFs ranged from 603 to 1113 . The linear range was $0.02-200 \_\mathrm{gL}^{-1}$ and the detection limit (DL) was $0.007-0.030 \_\mathrm{gL}-1$ for most of the analytes. [26] developed a new method for the extraction of organophosphorus pesticides (OPPs) from water samples by DLLME-GC-FPD. In this method, a mixture of $12.0 \_\mathrm{L}$ chlorobenzene and $1.00 \mathrm{~mL}$ acetone was rapidly injected into the $5.00 \mathrm{~mL}$ water sample by syringe. After centrifugation, 0.5 L of sedimented phase was injected into the GC. Under the optimum conditions, the PFs and extraction recoveries were obtained as $789-1070 \%$ and 78.9 $107 \%$, respectively.

Comparison of DLLME with SPME and SDME for the extraction of OPPs from water samples showed that DLLME is a very simple and rapid method (extraction time is less than $3 \mathrm{~min}$ ) and has high PF and extraction recoveries [56]. Described DLLME combined with GC-ECD for determining chlorobenzene (CBs) in water samples. The results indicated that DLLME is a sensitive, rapid and reproducible technique that can be used for preconcentration CBs from water samples. DLLME-GC-ECD also has been used for determination of trihalomethane (THMs) in drinking water [57]. Relative recoveries from the samples of drinking water spiked at the levels of 2.00 and 5.00_gL-1 were 95.0-107.8\%and 92.2-100.9\%, respectively. [58] Used DLLME in combination with gas chromatography-ion trap mass spectrometric detection (GC-MS) to preconcentrate triazine herbicides in water and the DLs in the range of $0.021-0.12 \mathrm{gL}-1$ were obtained. For the strong polar and nonvolatile samples, which are unsuitable for analysis by GC, derivatization is necessary to increase the analytes volatility. Application of DLLME coupled with derivatization reaction provides a one-step derivatization and extraction technique, greatly simplifying the operation steps and shortening the analysis time.

The GC-MS combined with DLLME to determine the anilines in wastewater samples [59]. In this method, the anilines are extracted by DLLME and derivatized using pentafluorobenzaldehyde (PFBAY) in aqueous solution simultaneously. Simultaneous derivatization and extraction by DLLME combined with GC-ECD was 
developed to determine chlorophenols (CPs) in water samples [60]. In this derivatization/extraction method, 500_L of acetone containing 10.0_L chlorobenzene and 50_L of acetic anhydride (derivatization reagent) was rapidly injected by syringe into $5.00 \mathrm{~mL}$ of aqueous sample containing CPs (analyte) and $\mathrm{K} 2 \mathrm{CO} 3(0.5 \%$, w/v). Within few seconds, the analytes were derivatized and extracted into the extracting solvent. Two methods based on HF-LPME and DLLME have been critically compared for the analysis of organo sulfur pesticides (OSPs) in environmental and beverage samples by GC-FPD [61]. Compared with HF-LPME, the advantages of DLLME were short extraction time and suitability for simultaneous treatment of batches of samples. Inaddition, a higher extraction recovery was obtained by DLLME in comparison with HF-LPME [61] However, when dealing with more complicated matrixes such as soil and beverage samples, HF-LPME was demonstrated to be more robust and sensitive than DLLME without sample filtration and dilution. Also the repeatability of HFLPME was better than that of DLLME.

Besides DLLME-GC combined with various detectors was applied to determine, phthalate esters in water samples[62], organ phosphorus flame retardants and plasticizers in water samples (NPD)[63], volatile phenols in red wines (MS) [64], speciation of butyl and phenyl compounds in water samples after derivatization with sodium tetraethyl borate(NaBEt4) (FPD) [65], amide herbicides in environmental water samples (MS) [66]. amitriptyline and nortriptyline in environmental sample solutions (FID) [76]. polychlorinated biphenyls in water samples (ECD) [68] fatty acids in water samples after derivatization with ethyl chloroformate (FID) [69], Rose extract constituents(MS) [70], phorate in water samples (FID) [71], parathyroid pesticide residues in water samples (ECD) [72], nitro aromatic compounds in water samples (FID) [73], methyl tert-butyl ether in water samples (FID) [74], personal care products in natural water samples (MS) [75], organ chlorine pesticides in water samples (MS) [76] and after conversion of calcium stearate to stearic acid in a polymeric matrix [77]. A solution of hydrochloric acid in 2-propanol was used to extract calcium stearate from its matrix. DLLME was applied to preconcentrate stearic acid before its injection in to GC instrument. DLLME-GC-MS/MS was used to determine triclosan (TCS) and methyltriclosan (MTCS) after derivatization N-methyl-N(tert-butyldimethylsilyl) trifluoroacetamide(MTBSTFA) [78].

\subsubsection{DLLME combined with HPLC}

In general, HPLC is a widely used versatile separation and quantification instrument. It is important that the selected extracting organic solvent for DLLME method be compatible with the HPLC mobile phase. However, halogenated hydrocarbons such as chlorobenzene, carbon tetrachloride, chloroform and tetrachloroethylene, usually selected as extracting solvents in DLLME, are not compatible with the reverse-phase-HPLC mobile phase, because of their high density and an extra step is needed to evaporate them before final analysis.

In 2007 [79] reported a preliminary study on a combination of DLLME with high-performance liquid chromatography-diode array detection (HPLC-DAD) for the analysis of antioxidants in aqueous samples. The reported method was very efficient, rapid and repeatable. Further, nearly $100 \%$ recovery and PFs about 200 times were attained. DLs of the method were between 3 and 7 ngmL $^{-1} \cdot[\mathbf{8 0}]$ applied DLLME in combination with HPLCvariable wavelength detection (VWD) for determination of methomylin water samples. Comparison of this method with SPE, SPME and SDME indicates that DLLME combine with HPLC-VWD is a simple, fast and low cost method thus it has tremendous potential in trace analysis of methomyl in natural waters.. [81] Employed DLLME combined with ultra-high pressure liquid chromatography (UHPLC)-tunable ultraviolet detection (TUV) for preconcentration and determination of triclosan (TCS), triclocarban (TCC) and methyltriclosan (M-TCS) in aqueous samples. Under the optimum conditions, linearity of the method was in the range of 0.05-100_gL-1 for TCS, 0.025-50.0_gL-1 for TCC and 0.500-100_gL-1 for M-TCS. DLs were in the range of 45.1-236 ng L-1. Recently, a novel method has been developed [82] for determining of biphenyl A in water samples by DLLMEHPLC- UV. The method showed an acceptable DL $(0.07$ gL-1) and good linear range (0.5-100_gL-1) without using any derivatization reagent or applying very sensitive determination methods as GC-MS and HPLCMS [83] described DLLMEHPLC- UV-vis detection for determination of met crate in water samples. Firstly, an orthogonal array design (OAD) was used to choose the significant factors. Secondly, the significant factors were optimized by a central composite design (CCD). Then, the quadratic model between the dependent and independent variables was built. The method showed a good agreement between the experimental data and predictive value[84].proposed DLLME combined with HPLC-UV for determining of Irgafos 1010 and Irgafos 168 from polyolefins. Then, acetonitrile $(2 \mathrm{~mL})$ and carbon tetrachloride $\left(200 \_\mathrm{L}\right)$ were added. The tube was capped and the mixture was heated at $100{ }^{\circ} \mathrm{C}$ in a water bath for $3 \mathrm{~h}$. After cooling and filtering, water $(5.00 \mathrm{~mL}) \mathrm{was}$ injected rapidly into the solution by a $5-\mathrm{mL}$ syringe. The cloudy solution produced was centrifuged for $5 \mathrm{~min}$ at $1000 \mathrm{rpm}$.

The sedimented phase was quantitatively transferred into another test tube and left to evaporate at room temperature. Finally, the residue was dissolved in $0.5 \mathrm{~mL}$ LC-grade methanol and 20_L of the obtained solution was then injected into HPLC for analysis. DLLME-HPLCDAD detection was used for extraction and preconcentration of phenoxyacetic acid herbicides in water samples [85]. A 5.00-mL water sample $(\mathrm{pH}=1.5)$ containing $10 \%(\mathrm{w} / \mathrm{v})$ sodium chloride was placed in a $10-\mathrm{mL}$ glass tube with a conical bottom. Acetone $(1 \mathrm{~mL})$ 
as disperse solvent and 25_L chlorobenzene as extracting solvent was injected rapidly into the sample. The mixture was then centrifuged for $5 \mathrm{~min}$ at $5000 \mathrm{rpm}$.

The method had good linearity and a wide linear dynamic range $(0.5-750$ gL-1). Further, its DL was 0.16 gL-1 for both analytes. [86] applied DLLME-HPLC-DAD for extraction and determination of ethylenediaminetetraaceticethylenediaminetetraacetic acid (EDTA) in sediments and water samples. A 7.00-mL working standard solution containing $30 \mathrm{ngmL}-1$ EDTA $(\mathrm{pH} 2.0)$ was placed in a 12-mL glass tube with conical bottom. Acetone (500_L) containing 50_L carbon tetrachloride was injected rapidly into the sample solution. After centrifuging, the sediment phase was completely transferred into another test tube with conical bottom using a 100- L HPLC syringe. After evaporation of the solvent in a water bath, the residue was dissolved in $50 \mathrm{~L}$ acetate buffer and injected into the separation system. Under the optimal conditions, the analytical range was 3.050.0_gL-1 and DL of 1.7_gL-1 was obtained for EDTA[87].reported DLLME combined with HPLC for determining benomyl in water samples. The method is based on the extraction of benomyl from acidified sample solution and its conversion into carbendazim via solvolysis reaction with $\mathrm{N}, \mathrm{N}$-dimethyl formamide (DMF) as organic solvent. It showed a good linearity $(0.998)$ with wide linear dynamic range $(0.01-25 \mathrm{mgL}-1)$ and low DL $(0.0033 \mathrm{mgL}-1)$. In addition, DLLME-HPLC in combination with different detectors was applied for determination of pentachlorophenol (DAD) [88].

\subsubsection{DLLME combined with AAS}

The majority of work on DLLME mentioned here has so far been focused on organic compounds, but there have been attempts to extend the procedure to inorganic analytes as well. Electro thermal atomic absorption spectrometry (ET-AAS) needs micro amounts of the sample for analysis. Therefore, by combination of DLLME and ET-AAS, a unique analysis system can be obtained. In this method, a chelating agent is added to the sample solution. Then, DLLME is conducted by using appropriate extracting and disperser solvents.. The application of this approach has been demonstrated for determination of cadmium in water samples[89]. Five hundred $\mu$ L of methanol containing 34 $\mu \mathrm{L}$ carbon tetrachloride and $0.00010 \mathrm{~g}$ of ammonium pyrrolidine dithiocarbamate was rapidly injected by a syringe into the water sample containing cadmium ions. After centrifugation (2 min at 5000 rpm), the droplets were sedimented at the bottom of the conical test tube $\left(25 \pm 1 \_L\right)$. Then, $20 \mu \_L$ of the sedimented phase containing enriched analyte was determined by ET-AAS. Under the optimum conditions, the PF was obtained for $\mathrm{Cd}$ is 25 using only $5.00 \mathrm{~mL}$ of the water sample. The calibration graph was linear in the range of $2-$ $20 \mathrm{ng} \mathrm{L}-1$ with the DL of $0.6 \mathrm{ng} \mathrm{L}-1$. The same authors also proposed DLLME combined with iridiummodified tube ET-AAS for determination of selenium (Se) in water samples [90]. The calibration graph was linear in the range of0.1-3 gL-1 with the DL of 0.05 gL-1.Later, the same authors established an optimal derivatizationreaction for Se(IV) followed by DLLME for micro extraction and analysis of the piazselenol complex formed by GC-ECD [91]. The results showed that to obtain a low DL value $(0.005$ gL- 1 , which was similar to that of the mass spectrometry detectors), DLLME requires the lower sample-preparation time and sample consumption $(5.00 \mathrm{~mL})$. Liang and Sang employed DLLME to determine the trace amount of lead in biological and water samples using ET-AAS.The DL of the proposed method for lead was obtained as $39 \mathrm{ng} \mathrm{L}-1$. The method was also applied for determination of $\mathrm{Pb}$ ions in human urine and tap water samples [92]. Extraction and preconcentration of $\mathrm{Cu}^{2+}$ ions by DLLME were optimized using one variable at a time as well as a simultaneous optimization method [93].

The optimization procedure was a chemo metric method using a central composite design for obtaining the optimal conditions. The application of DLLME has been extended to the selective determination of ultra trace amounts of gold in different samples [94]. The pre concentration procedure resulted in quantitative extraction of gold by Victoria Blue R from a 10-mL sample into fine droplets of chlorobenzene with a sediment volume of 25_L. The DL and relative standard deviation were $0.005 \mathrm{ngmL}^{-1}$ and $4.2 \%$, respectively.

In 2009, Rivas et al. [95] by combining DLLME with ET-AAS developed a new method for speciation of very low amounts of arsenic and antimony in water samples. The DLs of 0.01 and 0.05 gL-1 were obtained for $\mathrm{As}(\mathrm{III})$ and $\mathrm{Sb}(\mathrm{III})$, respectively, with the PF of 115. Besides, DLLME in combination with ET-AAS and FAAS were applied for speciation of $\mathrm{As}(\mathrm{III})$ and $\mathrm{As}(\mathrm{V})$ in water samples (ET-AAS) [96], preconcentration of palladium in water samples (ET-AAS) [97], selective determination of the trace amounts of palladium (FAAS) [98], rapid determination of lead in water samples (ET-AAS) [99], speciation of chromium in water samples (FAAS) [100],

\subsubsection{DLLME combined with spectrophotometric}

DLLME can also combine with spectrophotometric instruments for quantitative determination of metal ions. [101] proposed DLLME combined with fiber optical linear array detection spectrophotometer (FO-LADS) by using a cylindrical micro-cell for simultaneous pre concentration and determination of palladium and cobalt in real and synthetic samples. Under the optimum conditions, the calibration graphs were linear in the range of 2-100 and 1$70 \_\mathrm{gL}^{-1}$ with the DLs of $0.25 \_\mathrm{gL}^{-1}$ and $0.2 \_\mathrm{gL}^{-1}$ for palladium and cobalt, respectively. 


\subsection{Applications}

\subsubsection{Applications of DLLME for Environmental Water Samples}

DLLME has been successfully applied to extraction and concentration of a wide variety of organic compounds and metal ions, mainly from water of different types, such as tap, river, well and lake waters. Among them, pesticide analysis is probably the field in which DLLME has found its major applications. Substantial papers were devoted to development of DLLME for this group of analytes. Such a great number of papers concerning one group of analytes can be attributed to high interest in this field connected with food and environmental pollution caused by these compounds. Different methods based on DLLME were developed for the determination of different kinds of pesticide in water samples like tap water, lake water, and industrial waste water were established [102].

\subsubsection{Applications of DLLME for Soil and Sediment Samples}

Traditionally, DLLME is not suitable for soil samples. With the developments of DLLME combined with other extraction techniques, more and more papers have been published concerning about the applications of DLLME for soil and sediment samples. In the application to solid matrices, DLLME has acted as a cleaning and/or concentration step in the sample pre-treatment procedure. In some cases, the solvents used to extract the analytes from the solid matrix were utilized directly as the disperser in the DLLME process with good recoveries and clean sediment phases [103].

\subsubsection{Applications of DLLME for Food Samples}

Relatively few applications have been devoted to the analysis of organic compounds in highly complex matrices, such as food and biological samples, due to the interference of matrix components in these kinds of samples. Usually, the extracts of complex matrices may not be compatible with the DLLME process due to the interaction of matrix components with the extractants. An appropriate extractants should possess a higher extraction capacity for the analytes than the interferences. This higher extraction capacity is possible only if the analyte has a chemical behavior very different from the interferences. If raw extracts contain lipophilic matrix components, the hydrophobic nature of conventional DLLME extraction solvents is unable to discriminate among analytes and interferences, compromising the efficiency and selectivity of the process Two kinds of procedures were used for this purpose. In the first, the sample was homogenized and then centrifuged, or filtered juice was taken for DLLME. In the second group of procedures, samples were pre-extracted from the food matrix and the extract was used for DLLME

The QuEChERS technique (Quick, Easy, Cheap, Effective, Rugged, and Safe) was used several times in this field [30] About food samples, it can be divided into two categories: fluid food and solid matrix food. Applications of DLLME on fluid food such as wine, juice, and milk are more than solid matrix food. Mono-varietal wines, based on IL-DLLME implemented in a flow analysis system are proposed by [104].

Moreover, DLLME using extraction solvents lighter than water combined with high performance liquid chromatography for determination of synthetic antioxidants in fruit juice samples was proposed. The designed method was successfully applied for the preconcentration and determination of the studied synthetic antioxidants in different fruit juice samples, and satisfactory results were obtained [105].For the determination of macrocyclic lactones in milk, DLLME followed by liquid chromatography with diode array detection and atmospheric pressure chemical ionization ion-trap tandem mass spectrometry was developed [106]

A multiclass and multi-residue method was optimized and validated for analysis of 19 pesticides of 16 chemical classes in greenhouse cucumber and tomato followed by high performance liquid chromatographytandem mass spectrometry (HPLC-MS/MS). In this study, DLLME technique was applied for extraction and preconcentration of pesticide residues from QuEChERS (quick, easy, cheap, effective, rugged and safe) extracts [107].

\subsubsection{Applications of DLLME for Biological Samples}

A few papers have reported the application of DLLME on determination of drug mainly in urine. For the first time, DLLME-SFO was combined with field-amplified sample injection in capillary electrophoresis (CE) to determine four 2-agonists including cimbuterol, clenbuterol, mabuterol, and mapenterol in bovine urine. The applicability of the proposed method was successfully confirmed by determination of the four 2-agonists in spiked bovine urine and accuracy higher than $96.0 \%$ was obtained. The combination of DLLME and CE was particularly attractive because of the small amount of organic solvents required 108]

An environmentally friendly IL-DLLME method coupled with HPLC for the determination of antihypertensive drugs irbesartan and valsartan in human urine was developed 109]

In another study, DLLME followed by HPLC with diode array detection has been developed as sample preparation method for simultaneous determination of seven organic UV filters in urine. The proposed method was successfully applied to different volunteer urine samples and it was shown that the extraction efficiency was not affected by the type of urine samples [110].

Furthermore, a novel surfactant-assisted dispersive liquid-liquid micro extraction (SADLLME) based on SFO combined with HPLC-UV has been proposed for extraction and determination of amphetamine and 
methamphetamine in urine [111].In another study

Cypermethrin (CYP) insecticide in rat tissues (kidney, liver and brain) and blood has been developed for the first time using low density solvent DLLME followed by gas chromatography-electron capture detector (GC-ECD) analysis. Firstly, tissue samples containing CYP were homogenized in acetone. Subsequently, homogenate was mixed with n-hexane (extraction solvent) and the mixture was rapidly injected into water. Afterward, the upper nhexane layer was collected in a separate micro tube and then injected into GC-ECD for analysis. Blood samples were diluted with ultrapure water and subjected to DLLME through similar procedure. The results showed that this method is a simple, rapid and efficient technique for extraction and determination of CYP in rat tissues and blood [112]

\section{Conclusion}

DLLME has emerged as a viable sample-preparation approach with the advantages of simplicity of operation, rapidity, low cost, high recovery, and high pre concentration factor. Since it was invented in 2006, many improvements have been introduced such as conjunction with different extraction technique. Meanwhile, the application of DLLME has been expanded to some complex matrices samples such as environmental, food and biological samples, which not be confined to water samples. In the future, connection of DLLME to other extraction techniques is still the trend as they allow obtaining both better selectivity and lower limits of detection.

\section{Reference}

1. . Xu, L .C. Basheer, H.K.; Lee, J. Chromatogr. 2007, 1152. 184

2. Pena-Pereira, I. F.; Lavilla, C.; Bendicho. Spectro chim. Acta, Part B . 2009, 64

3. Arthur, C. L.; Pawliszyn. J, .J .Anal. Chem. 1990, 62 , 2145

4. . Liu, P.K.; Dasgupta, Anal. Chem. $1996,68,1817$

5. Jeannot, M.A.; Cantwell, F.F.J. Anal. Chem. 1996, 68 , 2236

6. Rasmussen, K.E.; Pederson-Bjergaard, S. Trends Anal. Chem.2004,23

7. . Psillakis, M.A.; Kalogerakis E. N. Trends Anal. Chem. 2002,21, 53

8. . Jeannot, F.F.; Cantwell, Anal. Chem. 1997, 69 , 235.

9. Ahmadi, F.Y.; Assadi, M.R.; Milani Hosseini, M.; Rezaee. J. Chromatogr. 2006, 1101, 307

10. Shen, G.; Lee, H.K. Anal. Chem. 2002, 74, 648

11. Pedersen-Bjergaard, K.E.; Rasmussen. Anal. Chem. 1999,71, 2650.

12. Silva, M.F.; Cerutti, E.S.; Martinez, Microchim. L.D. Acta 2006, 155,349.

13. . Carabias-Martinez, R. E.; Rodriguez-Gonzalo, B.; Moreno-Cordero, J.L.; PerezPavon, C.C.; Garcia-pinto, E.; Fernandez-Laespada. J.Chromatogr. 2000,902, 251.

14. . Ferrer, R .; Beltran, J.L.; Guiteras, J Anal. Chim. Acta . 1996, 330, $199 .$.

15. . Casero, I. D.; Sicilia, S.; Rubio, D.; Perez-Bendito. Anal. Chem. 1999, 17, 4519.

16. . Sicilia, D. S.; Rubio, D.; Perez-Bendito, N.; Maniasso, E.A.; Zagatto, G. Anal. Chim.Acta 1999, 29, 392

17. Takagai, Y. S.; Igarashi, Am. Lab. 2002, 34,30.

18. Oshite, S. M.; Furukawa, S.; Igarashi, Analyst.2001, 126, 703.

19. Igarashi, S. A.; Takahashi, Y.; Ueki, H.; Yamaguchi, Analyst.2000, 125,797

20. Khalili-Zanjani, M.R.; Yamini, Y. S.; Shariati, J.A.; Jonsson. Anal. Chim. Acta. 2007,585, 286

21. . Khalili-Zanjani, M .R.; Yamini, Y N.; Yazdanfar, S.; Shariati. Anal. Chim. Acta 2008, 606,202.

22. Regueiro, M. L.; Iompart, C.; Garcia-Jares, J.C.; Garcia-Monteagudo, R.; Cela. J. Chromatogr. 2008 ,1190, 27.

23. Luque de Castro, M. D.;Priego-Capote.;F: Analytical Applications of Ultrasound Elsevier: Amsterdam,2006.

24. Luque de Castro, M.D.; Priego-Capote, F: Talanta, 2007

25. . Rezaee, M .Y. Assadi, M.R. Milani Hosseini, E. Aghaee, F. Ahmadi, S. Berijani, J. Chromatogr. 2006, 1116

26. . Berijani, S. Y. Assadi, M. Anbia, M.R. Milani Hosseini, E. Aghaee, J. Chromatogr. 2006, 1123,1

27. Bidari, A.; Ganjali, M .R.; Assadi, Y. P.; Norouzi, A. novel methodology based on solvents less dense than water through dispersive liquid-liquid microextraction: application in quantitation of L-ascorbate in fruit juices and soft drinks by fiber optic-linear array detection, spectrophotometric. Anal. Methods . 2011, 3, 724730.

28. Guo, L.; Lee.; H.K. J.Chromatogr.2013,1300, 24.

29. Rezaee, M.Y.; Assadi, M.R.; Milani Hosseini, E.; Aghaee, F.; Ahmadi, S.; Berijani, Determination of organic compounds in water using dispersive liquid-liquid microextraction, J. Chromatogr. 2006, 1116, 1-9.

30. Zgola, A.; Grzeskowiak,T. Dispersive liquid-liquid microextraction. tract,rends Anal. Chem., 2011, 30,13821399

31. Rezaee, M.Y.; Yamini, M.; Faraji, Evolution of dispersive liquid-liquid micro extraction method, $J$. Chromatogr. $2010,1217,2342-2357$.

32. Rezaee , M .; Yamini, Y .; Faraji, M. Evolution of dispersive liquid-liquid microextraction method . J. 
Chromatogr.2010 1217: 2342-2357.

33. Li, Z. L.; Chen, F.; Wang, X. D.; Wang, C. J. Ionic liquids dispersive liquid-liquid microextraction and highperformance liquid chromatographic determination of irbesartan and valsartan in human urine. Biomed. Chromatogr.2013, 27, 254-258

34. Li, T. H.; Joshi, M. D.; Ronning, D. R.; Anderson, J. L. Ionic liquids as solvents for in situ dispersive liquidliquid microextraction of DNA. J. Chromatogr. 2013, $1272,8-14$..

35. Anthemidis, A. N.;Ioannou.; K. I. G. Sequential injection ionic liquid dispersive liquid-liquid microextraction for thallium preconcentration and determination with flame atomic absorption spectrometry. Anal. Bioanal. Chem. 2012 ,404, 685-691

36. Asensio-Ramos, M.; Hernandez-Borges, J.; Ravelo-Perez, L. M.; Afonso, M. M.; Palenzuela, J. A.; Rodriguez-Delgado, M. A. Dispersive liquid-liquid microextraction of pesticides and metabolites from soils using 1,3-dipentylimidazolium hexafluorophosphate ionic liquid as an alternative extraction solvent. Electrophoresis, 2012,33, 1449-1457.

37. .Zhao, A. J.; Wang, X. D.; Ma, M. P.; Wang, W. W.; Sun, H. L.;Yan, Z. J.; Xu, Z. T.; Wang, H. L. Temperature-assisted ionic liquid dispersive liquid-liquid microextraction combined with high performance liquid chromatography for the determination of PCBs and PBDEs in water and urine samples. Microchim. Acta . 2012, 177, 229-236.

38. Zhang, Z.; Zhou, K.; Bu, Y. Q.; Shan, Z. J.; Liu, J. F.; Wu, X. Y.; Yang, L. Q.; Chen, Z. L. Determination of malachite green and crystal violet in environmental water using temperature-controlled ionic liquid dispersive liquid-liquid microextraction coupled with high performance liquid chromatography. Anal. Methods .2012, 4, 429-433.

39. .Vazquez, M. D. P.; Vazquez, P. P.; Galera, M. M.; Sanchez, L. M. Simple, rapid, and sensitive determination of beta-blockers in environmental water using dispersive liquid-liquid microextraction followed by liquid chromatography with fluorescence detection. J. Sep. Sci. 2012 35,2184-2192

40. .Zhang, Y. F.; Lee, H. K. Determination of ultraviolet filters in water samples by vortex-assisted dispersive liquid-liquid microextraction followed by gas chromatography-mass spectrometry. J. Chromatogr. 2012, 1249, 25-31.

41. Naeeni, M. H.; Yamini, Y.; Rezaee M. Combination of supercritical fluid extraction with dispersive liquidliquid microextraction for extraction of organophosphorus pesticides from soil and marine sediment samples. J. Supercrit. Fluids, 2012, 57, 219-226

42. .Ghambari, H.; Hadjmohammadi, M. Low-density solvent-based dispersive liquid-liquid microextraction followed by high performance liquid chromatography for determination of warfarin in human plasma . J. Chromatogr. 2012, 899, 66-71

43. .Prichodko, A.,Janenaite, E.; Smitiene, V.; Vickackaite, V.Gas chromatographic determination of parabens after in-situ derivatization and dispersive liquid-liquid microextraction. Acta .Chromatogr.2012, 24, 589-601

44. .Ranjbari, E.; Biparva, P.; Hadjmohammadi, M. R. Utilization of inverted dispersive liquid-liquid microextraction followed by HPLC-UV as sensitive and efficient method for the extraction and determination of quercetin in honey and biological samples ; Talanta , 2012.

45. Zhang, J. H.; Liang, Z.; Li, S. Q.; Li, Y. B.; Peng, B.; Zhou, W. F.; Gao, H. X. In-situ metathesis reaction combined with ultrasound-assisted ionic liquid dispersive liquid-liquid microextraction method for the determination of phenylurea pesticides in water samples; Talanta ,2012.

46. .Rezaee, M.; Yamini, Y.; Faraji, M. Evolution of dispersive liquid-liquid microextraction method . J. Chromatogr. 2010 ,1217: 2342-2357

47. .Rezaee, M.; Mashayekhi, H. A. Solid-phase extraction combined with dispersive liquid-liquid microextraction as an efficient and simple method for the determination of carbamazepine in biological samples . Anal. Methods .2012, 4: 2887-2892

48. Mohammadi, S. Z.; Baghelani, Y. M.; Mansori, F.; Shamspur, T.; Afzali, D. Dispersive liquid-liquid microextraction for the simultaneous separation of trace amounts of zinc and cadmium Ions in water samples prior to flame atomic absorption spectrometry determination. Quimica . Nova. 2012 , 35, 198-202

49. Xiong, J. F.; Guan, Z.; Zhou, G. M.; Tang, X. Y.; Lv, Y. J.; Wang, H. L. Determination of chlorpyrifos in environmental water samples by dispersive liquid-liquid microextraction with solidification of a floating organic drop followed by gas chromatography with flame photometry detection. Anal. Methods .2012,4, 32463250

50. Tehrani, M. S.;Givianrad, M. H.; Mahoor, N. Surfactant-assisted dispersive liquid-liquid microextraction followed by high-performance liquid chromatography for determination of amphetamine and methamphetamine in urine samples. Anal. Methods. 2012 , 4, 1357-1364.

51. Rezaee, M.; Assadi, Y.; Hosseinia, M. R. M.; Aghaee, E.;Ahmadi, F.; Berijani, S. Determination of organic compounds in water using dispersive liquid-liquid microextraction. J. Chromatogr.2006,1116, 1-

52. Zgola, A ; Grzeskowiak, T. Solid-phase extraction combined with dispersive liquid-liquid microextraction, 
fast derivatization and high performance liquid chromatography-tandem mass spectrometry analysis for trace determination of short-chained dodecyl alcohol ethoxylates and dodecyl alcohol in environmental water samples. J. Chromatogr. 2012 , 1251, 40-4

53. Li, Y.; Liu, J. L.; Zhang, C.; Wang, X. J. Combination of solid phase extraction-dispersive liquid- liquid microextraction and pre-column derivatization method for determination of trace level estrogens in water sample. Chin. J. Anal. Chem.2012 40: 107-112.

54. Rezaee, M.; Yamini, Y.; Moradi, M.; Saleh, A.; Faraji, M.; Naeeni, M. H.. Supercritical fluid extraction combined with dispersive liquid-liquid microextraction as a sensitive and efficient sample preparation method for determination of organic compounds in solid samples. J. Supercrit. Fluids. 2010 , 55,161-168

55. . Kozani, R.R.; Assadi, Y. F.; Shemirani, M. R.; Milani Hosseini, M. R. Jamali: Talanta, 72, 2007.

56. Nagaraju, D .; Huang, S.D . J. Chromatogr. 2007, 1161,89

57. Chiang, J.S.; Huang, S.D. Talanta . 2008, 75,70

58. Fattahi, N.Y.; Assadi, M.R.; Milani Hosseini, E.Z.; Jahromi . J. Chromatogr. 2007 , 1157, 23

59. Xiong, J. B.; Hu . J. Chromatogr. $2008,1193,7$.

60. Farahani, H. P.; Noruzi, R.; Dinarvand, M. R.; Ganjali. J. Chromatogr. 2007,1172, 105.

61. . Garcia-Lopez, M. I.; Rodriguez, R. Cela, J. Chromatogr. 2007,1166, 9

62. Farina,L. E.; Boido, F.; Carrau, E.; Dellacassa. J. Chromatogr. 2007,1157, 46

63. Pajand Birjandi, A. A.; Bidari, F.; Rezaei, M.R.; Milani Hosseini, Y.; Assadi. J. Chromatogr. 2008, $1193,19$.

64. .Zhao, R.S.; Diao, C.P.; Wang, X. T.; .Jiang,J.P.; Yuan. Anal.Bioanal.Chem. 2008, 391.

65. Sarafraz-Yazdi, A. N.; Razavi, S. Raouf Yazdinejad. Talanta .2008, 75, 1293.

66. .Rezaei,F.A.;Bidari,A.PajandBirjandi,M.R.;MilaniHosseini,Y.;Assadi.J.Hazard. Mater. 2008, $158,621$.

67. . Pusvaskiene, E, B.; Januskevic, A.; Prichodko, V.; Vickackaite. Chromatogra 2009, 69, 271.

68. Sereshti, H. M.; Karimi, S.; Samadi. J. Chromatogr. 2009, 1216,198.

69. Hong-Xue, X. H.; Li-Jun, W.; Xiu-Ling, F.; Lu, L.; Kui, W.; Mei-Mei, M.; Da-Wei, Chin. J.Anal.Chem. 2008, 36,1543

70. Xiao-Huan, Z. W.; Chun, G.; Shu-Tao, Z.; Xin, W,; Zhi, Chin. J. Anal. Chem. 2008, 36, 765.

71. Ebrahimzadeh, H. Y.; Yamini, F. Kamarei, Talanta, 2009 79, 1472

72. Farhadi, K. R.; Maleki, N.; Mohammad Nezhad. J. Chin. Chem. Soc.56 2009, 56. 575.

73. Panagiotou, A.N.; Sakkas, V.A.; Albanis, T.A. Anal. Chim. Acta. 2009, 649, 135.

74. Cortada, C. L.; Vidal, R.; Pastor, N.; Santiago, A.; Canals. Anal. Chim. Acta 2009, 649, 218.

75. Ranji, A.; Ravandi, M. G.; Farajzaded, M . A. Anal. Sci. 2008, 24,623.

76. Montes, R. I.; Rodriguez, E.; Rubi, R.; Cela. J. Chromatogr. 2009, 1216,205.

77. . Farajzadeh, M. A.; Bahram, M.; Jonsson. J.A. Anal. Chim. Acta. 2007, 591,69

78. Wei, G. Y.; Wang, Li, X. J. Sep. Sci. 2007, 30, 3262.

79. Guo,X.H.;Li,X.L.;Cao,Y.;Li,X.Z.;Wang,X.B.;Xu.J.Chromatogr.2009,1216, 3038

80. Rezaee, M. Y.; Yamini, S.; Shariati, A.; Esrafili, M.; Shamsipur. J. Chromatogr. 2009, 1216, 1511.

81. Xia, J .; Xiang, B.; Zhang, W.; Anal. Chim. Acta 2008 625,28.

82. Farajzadeh, M.A.; Vardast, M .R.; Bahram M. Chromatographia 2009, 69,409.

83. Farhadi, K.; Matin, A.A.; Hashemi, P. Chromatographia 2009, 69,45.

84. Maleki, R. N.; Mohammad Nezhad, N.; Samadi, K.; Farhadi, Microchim. Acta 2009, 165,97.

85. Farhadi, K.; Farajzadeh, M.A.; Matin, A.A. J. Sep. Sci. 2009, 32,2442

86. .Farhadi,K.;Farajzadeh,M.A.;MatinA.A.;.Hashemi,P.Cent.Eur.J.Chem. 2009, 7, 369.

87. Zeini Jahromi, E. A.; Bidari, Y.; Assadi, M.R.; Milani Hosseini, M.R.; Jamali. Anal. Chim.Acta. 2007, 585,305 .

88. Bidari, A. E.; Zeini Jahromi, Y.; Assadi, M.R.; Milani Hosseini. . J. Micro chem 2007, 87.

89. Bidari, A. P.; Hemmatkhah, S.; Jafarvand, M.R.; Milani Hosseini, Y.; Assadi. Microchim.Acta. 2008, 163,243.

90. Liang, P. H.; Sang. Anal. Bio chem. 2008, 380, 21.

91. Farajzadeh, M.; Bahram, B.; Ghorbani Mehr, J.A.; Jonsson. Talanta 2008, 75,832.

92. .Shamsipur, M.; Ramezani, M. Talanta. 2008, 75, 294

93. Naseri, P.; Hemmatkhah, M. R.; Milani Hosseini, Y.; Assadi. Anal. Chim. Acta 2008, 610,135.

94. .Rivas, R.E.; Lopez-Garcia, I. M.; Hernandez-Cordoba, Spectrochim. Acta, Part B 2009,64,329

95. Shokoufi, N. F.; Shemirani, Y.; Assadi. Anal. Chim. Acta 2007,597,349.

96. .Liang, P. E.; Zhao, F.; Li, Talanta. 2009, 77, 1854

97. .Naseri, M.R.; Milani Hosseini, Y.; Assadi, A.; Kiani. Talanta, 2008

98. Hemmatkhah,P.A.; Bidari,S.; Jafarvand,M.R.; MilaniHosseini,Y.; Assadi. Micro chim.Acta 2009,166,69.

99. Shokoufi, N. F.; Shemirani, Y.; Assadi. Anal. Chim. Acta . 2007, 597.349.

100.Zuo, M..; Cheng, J.; Matsadiq, G.; Liu, L.; Li, M. L.; Zhang, M. Application of dispersive liquid-liquid microextraction based on solidification of floating organic droplet multi-residue method for the simultaneous determination of polychlorinated biphenyls, organochlorine, and pyrethroid pesticides in aqueous sample. 
Clean-Soil Air Water. 2012, 40, 1326-1333

101. Campone, L.;Piccinelli, A. L.;Celano, R.; Rastrelli, L. pH-controlled dispersive liquid-liquid microextraction for the analysis of ionizable compounds in complex matrices: Case study of ochratoxin A in cereals. Anal. Chim. Acta.2012, 754, 61-66

102.Escudero, L. B.; Martinis, E. M.; Olsina, R. A.; Wuilloud, R. G.; Arsenic speciation analysis in mono-varietal wines by on-line ionic liquid-based dispersive liquid-liquid microextraction. Food Chem, 2013, 138: 484-490.

103.Biparva, P.; Ehsani, M.; Hadjmohammadi, M. R). Dispersive liquid-liquid microextraction using extraction solvents lighter than water combined with high performance liquid chromatography for determination of synthetic antioxidants in fruit juicesamples .J. Food Compos. Anal. 2012,27,87-94.

104.Campillo, N.; Vinas, P.; Ferez-Melgarejo, G.; Hernandez-Cordoba, M. Dispersive liquid liquid microextraction for the determination of macrocyclic lactones in milk by liquid chromatography with diode array detection and atmospheric pressure chemical ionization ion-trap tandem mass spectrometry. $J$. Chromatogr. 2013 1282, 20-26

105. Adlnasab, L.; Ebrahimzadeh, H.; Yamini, Y. (A three phase dispersive liquid-liquid microextraction technique for the extraction of antibiotics in milk. Microchim. Acta. 2012, 179,179-184

106. Sereshti, H.; Samadi, S.; Jalali-Heravi, M). Determination of volatile components of green, black, oolong and white tea by optimized ultrasound-assisted extraction-dispersive liquid-liquid microextraction coupled with gas chromatography. J. Chromatogr. 2013,1280,1-8..

107.Zacharis, C. K.; Rotsias, I.; Zachariadis, P. G.; Zotos, Dispersive liquid-liquid microextraction for the determination of organo chlorine pesticides residues in honey by gas chromatography-electron capture and ion trap mass spectrometric detection. Food Chem. 2012,134,1665-1672

108. Dashtbozorgi, Z.; Ramezani, M. K.; Waqif-Husain, S.). Optimization and validation of a new pesticide residue method for cucumber and tomato using acetonitrile-based extraction dispersive liquid-liquid microextraction followed by liquid chromatography-tandem mass spectrometry. Anal. Methods, 2013, 5, 1192-1198.

109.Kohler, I.; Schappler, J.; Sierro, T.; Rudaz, S. J. Dispersive liquid-liquid microextraction combined with capillary electrophoresis and time-of-flight mass spectrometry for urine analysis. J.Pharm. Biomed. Anal., 2013, 73,82-89.

110.Li, Z. L.;Chen, F.; Wang, X. D.; Wang, C. J. Ionic liquids dispersive liquid-liquid microextraction and highperformance liquid chromatographic determination of irbesartan and valsartan in human urine. Biomed. Chromatogr. 2013 27, 254-258

111. Vosough, M.; Mojdehi, N. R.; Salemi, A. Chemometrics assisted dispersive liquid-liquid microextraction for quantification of seven UV filters in urine samples by HPLC-DAD .J.Sep.Sci.2012, 35: 3575-3585

112. Tehrani, M. S.; Givianrad, M. H.; Mahoor, N. Surfactant-assisted dispersive liquid-liquid microextraction followed by high-performance liquid chromatography for determination of amphetamine and methamphetamine in urine samples. Anal. Methods, 2012 4,1357-1364

113. Mudiam, M. K. R.; Jain, R.; Maurya, S. K.; Khan, H. A.; Bandyopadhyay, S.; Murthy, R. C. Low density solvent based dispersive liquid-liquid microextraction with gas chromatography-electron capture detection for the determination of cypermethrin in tissues and blood of cypermethrin treated rats. J. Chromatogr. 2012, $895,65-70$ 